\title{
Prognostic value of extracapsular invasion and fibrotic focus in single lymph node metastasis of gastric cancer
}

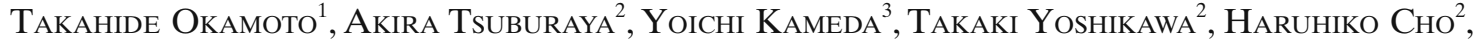 \\ Kazuhito Tsuchida ${ }^{2}$, Shinichi Hasegawa ${ }^{2}$, and Yoshikazu Noguchi ${ }^{1}$ \\ ${ }^{1}$ Department of Surgery, Shonan Tobu Sogou Hospital, 500 Nishikubo, Chigasaki 253-0083, Japan \\ ${ }^{2}$ Department of Gastrointestinal Surgery, Kanagawa Cancer Center, Yokohama, Japan \\ ${ }^{3}$ Department of Pathology, Kanagawa Cancer Center, Yokohama, Japan
}

\begin{abstract}
Background. Histological findings of metastatic lymph nodes are important prognosticators in patients with gastric cancer. The aim of this study was to clarify the clinical significance of various pathological characteristics of the early phase of lymph node metastasis in patients with gastric cancer, by selecting patients with tumors that had single lymph node metastases, no serosal invasion, and no metastases to the peritoneum, liver, or distant organs.

Methods. Seventy-eight patients were eligible and were entered in this study. These patients were subdivided according to the following histological characteristics of the one metastatic lymph node: size of the metastasis (i.e., amount of tumor cells $[\mathbf{A T}])$, proliferating pattern (PP), intranodal location (IL), and the presence or absence of extracapsular invasion (ECI) and/or fibrotic focus (FF). Associations between clinicopathological factors, survival, and the nodal findings were examined.

Results. There were no correlations between AT or PP and any clinicopathological factors. IL was significantly correlated with venous invasion and the pathological characteristics of the primary tumor. ECI and FF were observed significantly more frequently in pT2 than in pT1 cancer. Overall survival (OS) differed significantly according to depth of invasion, venous invasion, and the presence or absence of ECI or FF, although OS was not affected by AT, PP, or IL. The 10-year overall survival rates of patients with and without ECI were $50 \%$ and $80 \%$, respectively, while these rates for patients with and without FF were $50 \%$ and $79 \%$, respectively. Multivariate analysis revealed that ECI and FF were significant prognosticators of survival.

Conclusion. These results strongly suggested that the presence of ECI or FF could affect the survival of patients with gastric cancer.
\end{abstract}

Key words Extracapsular invasion - Lymph node metastasis · Gastric cancer

Offprint requests to: T. Okamoto

Received: August 28, 2007 / Accepted: May 20, 2008

\section{Introduction}

Histological complete resection (R0) of tumors is the only potentially curative treatment for patients withgastric carcinoma. The National Comprehensive Cancer Network (NCCN) recommends curative gastrectomy with systematic lymph node dissection up to second-tier nodes (D2), when tumors are confined to the primary lesion and regional lymph nodes. Nevertheless, localized disease frequently recurs after curative resection, even of T1 early tumors. It is difficult to anticipate the prognosis of patients who undergo curative surgery for early disease. So far, many biological and histological markers other than $\mathrm{T}$ and $\mathrm{N}$ have been reported and discussed as prognostic factors [1,2]. The histological findings of metastatic lymph nodes are among such prognosticators [3].

Numerous reports have assessed the correlation between the histology of lymph node metastasis and patients' prognoses in various types of tumors, including breast and colorectal tumors $[4,5]$. The presence of extracapsular invasion (ECI) has been reported to be a significant prognostic factor in patients with gastric cancer $[3,6]$. Recently, it has been suggested that perinodal involvement is related to advanced stage and appears to be a reliable prognostic factor for gastric cancer [7-9]. However, conclusive results have not been drawn from these previous reports because of their inclusion of heterogeneous tumor stages.

The prognosis of patients with gastric carcinoma correlates with the $\mathrm{T}$ and $\mathrm{N}$ stage, with minimum recurrence of T1N0 or T2N0 tumors being seen after resection. T3 tumors are predisposed to peritoneal dissemination, and the number of metastatic lymph nodes constitutes TNM staging. To determine the clinical significance of various pathological characteristics of metastatic lymph nodes, with a minimum confounding effect of peritoneal and other factors, a study population should ideally be limited to patients with a single 
lymph node metastasis without serosal invasion, peritoneal metastases, liver metastases, or distant metastases. In this study, we retrospectively studied patients who had undergone radical resection of $\mathrm{T} 1$ or $\mathrm{T} 2$ gastric cancer with a single lymph node metastasis. The pathology of the lymph node metastases was categorized according to the size of the metastasis (i.e., amount of tumor cells [AT]), the proliferating pattern (PP), intranodal location (IL), and the presence or absence of extracapsular invasion (ECI) and/or fibrotic focus $(\mathrm{FF})$ to elucidate the prognostic value of these nodal findings.

\section{Patients and methods}

\section{Patient selection}

Between 1979 and 1994, 1568 patients with primary gastric cancer underwent a gastrectomy with lymph node dissection at the Department of Gastrointestinal Surgery, Kanagawa Cancer Center, Yokohama, Japan. The resected specimens were examined, and findings were recorded according to the Japanese classification of gastric carcinoma, 2nd English edition, of the Japanese Gastric Cancer Association [10]. The postoperative outcomes were obtained through routine clinical appointments at the outpatient clinic or by telephone contact. Of these patients, we selected patients for this cohort study according to the following eligibility criteria: (a) adenocarcinoma of the stomach confirmed histologically; (b) pT1 or pT2 tumors (i.e., not exposed to the serosa); (c) no metastases to the liver, peritoneum, or distant organs; (d) patients who had undergone curative gastrectomy with complete or partial removal of the second-tier lymph nodes; (e) regional lymph node metastasis limited to a single node with a diameter greater than $0.2 \mathrm{~mm}$ (i.e., isolated tumor cells were excluded); (f) patients had not undergone either chemotherapy or radiotherapy before the operation.

A total of 78 patients were eligible and were entered in this study. The background of the patients is summarized as follows. There were 41 men and 37 women, with an age range of 43-80 years (mean, 60 years). There were two mucosal tumors, 29 submucosal, 23 muscular, and 24 subserosal tumors. Seven tumors had skip metastasis beyond perigastric lymph node stations. There were 46 differentiated-type and 32 undifferentiated-type tumors. The histology of differentiated type included well- or moderately differentiated tubular adenocarcinoma and papillary adenocarcinoma of the Japanese classification, whereas the undifferentiated type included poorly differentiated adenocarcinoma, signet-ring cell carcinoma, and mucinous adenocarcinoma.

\section{Pathological examination of the metastatic lymph nodes}

All pathological examinations were done by pair-review, with one pathologist (Y. K.) and one surgeon who had been trained as a trainer at the pathology section for more than 2 years (T. O.). All specimens were formalinfixed and paraffin-embedded, and several sections of the primary tumors were examined by hematoxylin and eosin (H\&E) and elastic fiber stainings. One cross section through the center of each lymph node was examined by H\&E staining. First, all the dissected lymph nodes were re-reviewed, and it was confirmed that the metastasis was confined to only a single node. After that, detailed histological characteristics of the metastatic lymph node were evaluated and classified according to the categories described below.

\section{Classification of the nodal findings}

The amount of tumor cells (AT) in the node was evaluated by measuring the size of the metastatic focus, using an eyepiece graticule, and categorized as a micrometastasis $(<2 \mathrm{~mm})$ or a macrometastasis $(\geq 2 \mathrm{~mm})$ by the largest diameter. The proliferating pattern (PP) of the tumor cells was classified as nodular, diffuse, or microfocal type (Arai et al. [11]; Fig. 1). The IL of the cancer focus was categorized as marginal sinus, medullary sinus, or mixed type (Natsugoe et al. [12]; Fig. 2) ECI was defined as positive when the tumor cells clearly extended beyond the nodal capsule into perinodal tissue (Fig. 3). Invasion into the perinodal lymph vessels was defined as positive. FF was defined as positive when the metastatic focus had abundant collagen-bundle-like scar areas, irrespective of whether the focus was inside or outside the node (Fig. 3) [13].

\section{Follow-up of the patients}

After the surgery, patients were followed up at the outpatient clinic every 3 months for the first 3 years, and then every 6 months for the subsequent 2 years. The patients were checked by physical examinations and assessed for serum levels of the tumor markers carcinoembryonic antigen (CEA) and carbohydrate antigen (CA19-9) at every visit, and biannual abdominal ultrasonography or computed tomography (CT) scanning was performed. When a recurrence of cancer was highly suspected, the patients underwent examinations using a combination of barium enema X-radiography, CT scanning, and/or diagnostic laparoscopy to determine the site of the recurrence.

\section{Statistical analyses}

The $\chi^{2}$ method was used for comparisons between two or among more than two groups. Survival was analyzed 


\section{Nodular Type}

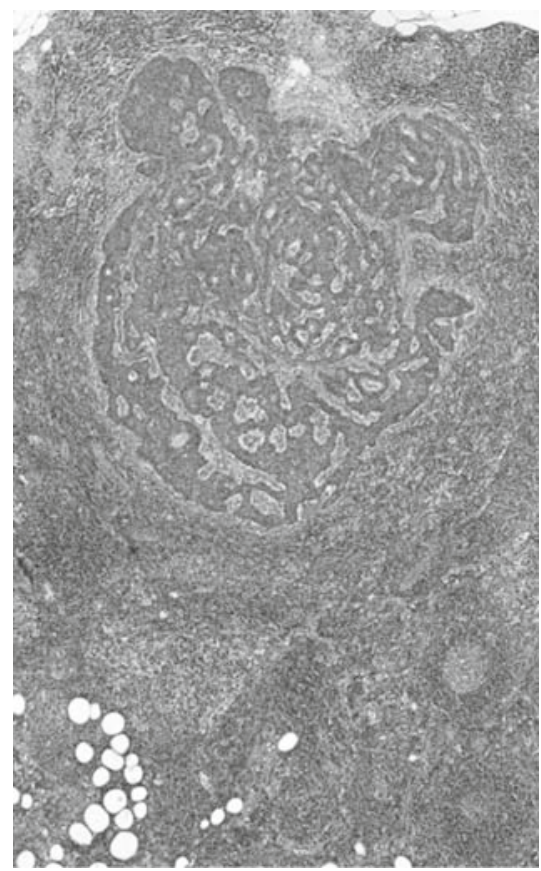

Diffuse type

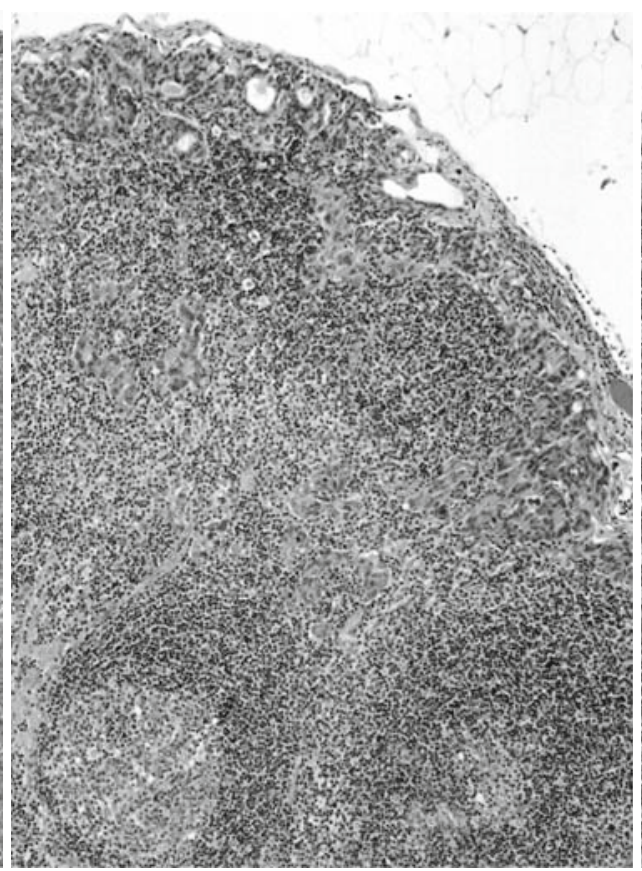

Micro focal type

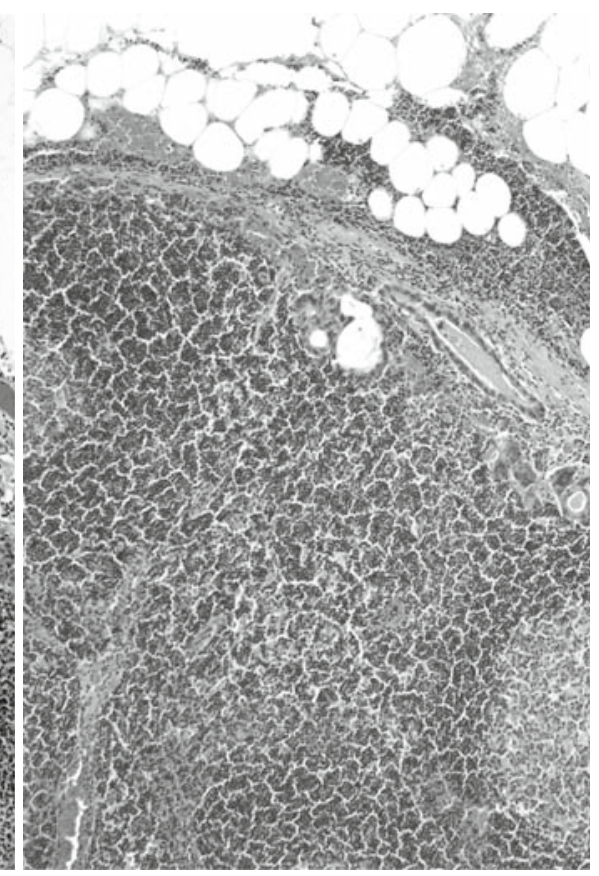

Fig. 1. Proliferating pattern of metastasis $(\mathrm{PP}), \mathrm{H} \& \mathrm{E}$

\section{Marginal sinus type}

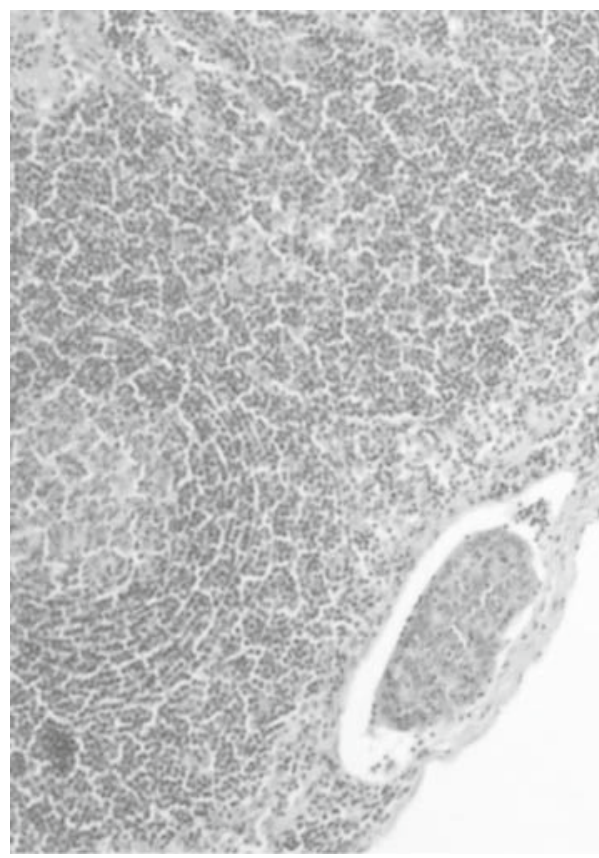

Fig. 2. Intranodal location (IL), H\&E
Medullary type

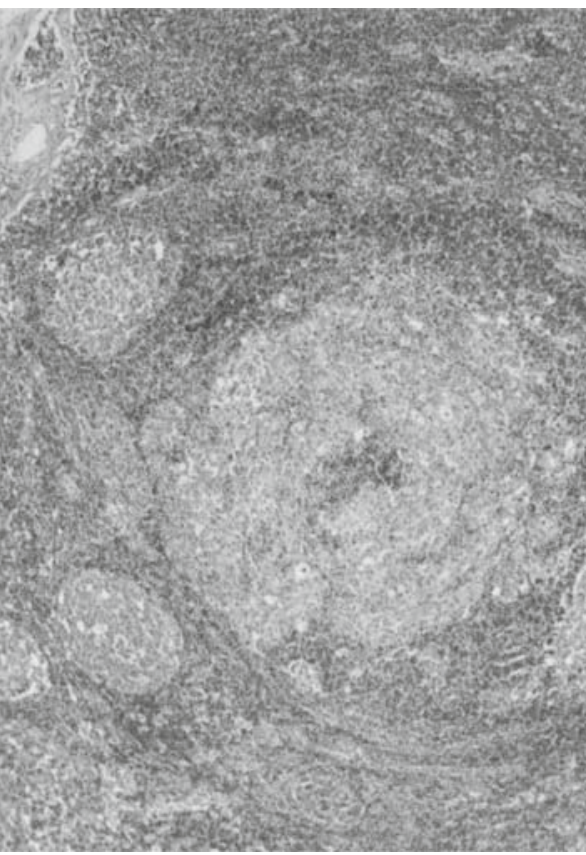

\section{Mixed type}

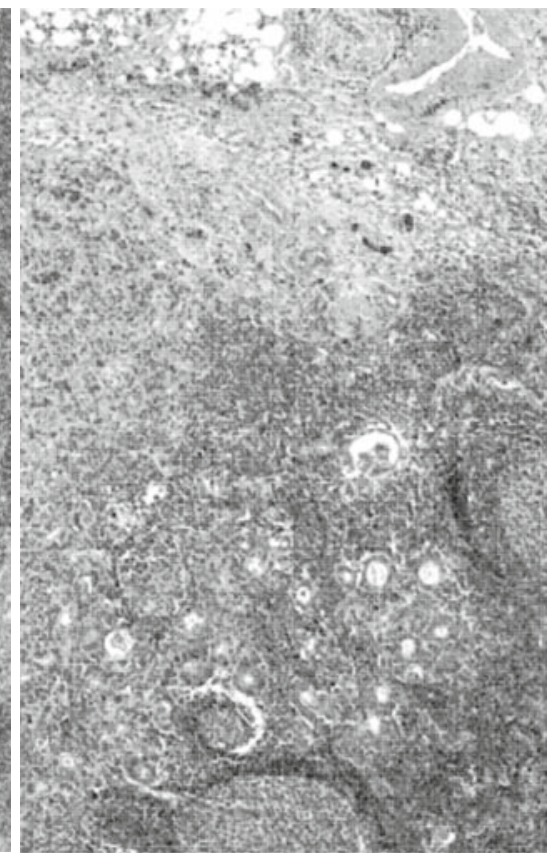


ECI

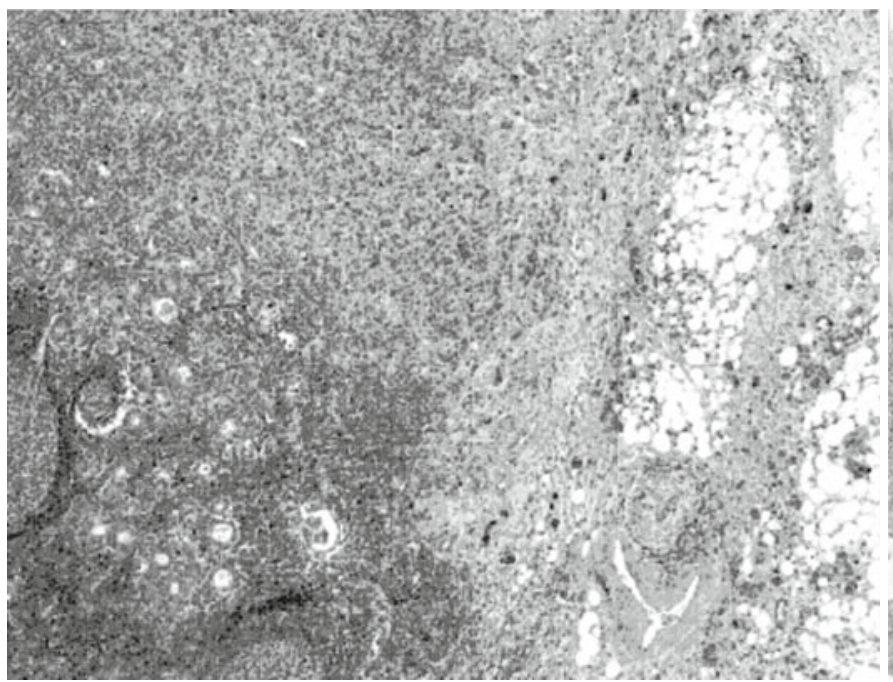

FF

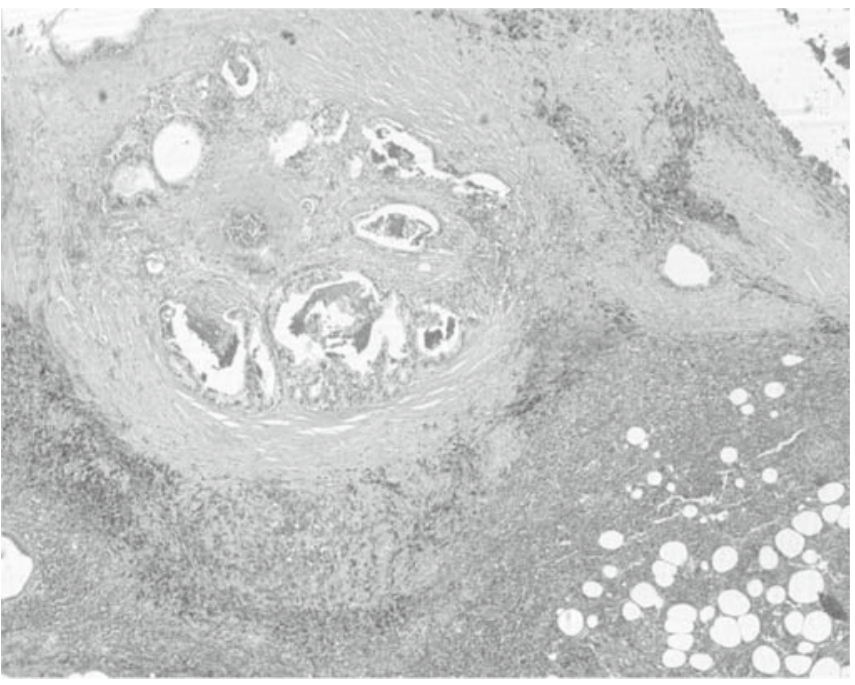

Fig. 3. Extracapsular invasion $(E C I)$ and fibrotic focus $(F F)$. ECI, tumor cells clearly extend beyond the nodal capsule into perinodal adipose tissue. Fibrotic focus consists of hyalinized collagen bundles with coagulative necrosis

Table 1. Correlation between size of metastasis (AT) and clinicopathological findings

\begin{tabular}{|c|c|c|c|}
\hline \multirow[b]{2}{*}{ Variable } & \multicolumn{2}{|c|}{ Size of metastasis (AT) } & \multirow[b]{2}{*}{$P$ value } \\
\hline & $\begin{array}{c}\text { Micrometastasis }(\leq 2 \mathrm{~mm}) \\
(n=34)\end{array}$ & $\begin{array}{c}\text { Macrometastasis }(>2 \mathrm{~mm}) \\
(n=44)\end{array}$ & \\
\hline \multicolumn{4}{|l|}{ Depth of tumor } \\
\hline pT1 & 14 & 17 & NS \\
\hline $\mathrm{pT} 2$ & 20 & 27 & \\
\hline \multicolumn{4}{|l|}{ Pathological type } \\
\hline Differentiated & 17 & 29 & NS \\
\hline Undifferentiated & 17 & 15 & \\
\hline \multicolumn{4}{|c|}{ Greatest primary tumor dimension } \\
\hline$\leq 30$ & 14 & 15 & NS \\
\hline$>30$ & 20 & 29 & \\
\hline \multicolumn{4}{|l|}{ Lymphatic invasion } \\
\hline None to mild & 27 & 40 & NS \\
\hline Moderate/Severe & 7 & 4 & \\
\hline \multicolumn{4}{|l|}{ Vascular invasion } \\
\hline Negative & 34 & 37 & NS \\
\hline Positive & 0 & 7 & \\
\hline
\end{tabular}

using the Kaplan-Meier method and log-rank test. Cox's proportional hazard model was used for multivariate analyses. $P$ values of less than 0.05 were considered statistically significant. All the statistical analyses were carried out using SPSS statistics software (SPSS, Chicago, IL, USA)

\section{Results}

Clinicopathological findings of the primary tumor and nodal findings. The median maximum diameter of the metastatic focus (AT) was $4.7 \mathrm{~mm}$ (range, $0.02-33 \mathrm{~mm}$ ).
There were no correlations between the clinicopathological characteristics of the tumor and the nodal findings of AT (Table 1). In regard to intranodal PP, 37 were nodular type, 29 diffuse type, and 12 were the microfocal type. No significant correlations were found between the clinicopathological findings and PP. In regard to IL, 34 were marginal, 3 medullary, and 41 mixed. IL was significantly associated with the histology of the primary tumor and venous invasion; tumors with undifferentiated histology or venous invasion tended to metastasize as mixed type. ECI and FF were more frequent in metastases from pT2 tumors than in metastases from pT1 tumors $(P=0.03$ and $P=0.008$, respectively; 
Table 2. Correlation between extracapsular invasion (ECI) and clinicopathological findings

\begin{tabular}{|c|c|c|c|}
\hline \multirow[b]{2}{*}{ Variable } & \multicolumn{2}{|c|}{ Extracapsular invasion (ECI) } & \multirow[b]{2}{*}{$P$ value } \\
\hline & Positive $(n=32)$ & Negative $(n=46)$ & \\
\hline \multicolumn{4}{|l|}{ Depth of tumor } \\
\hline pT1 & 8 & 23 & 0.03 \\
\hline pT2 & 24 & 23 & \\
\hline \multicolumn{4}{|l|}{ Pathological type } \\
\hline Differentiated & 19 & 27 & NS \\
\hline Undifferentiated & 13 & 19 & \\
\hline \multicolumn{4}{|c|}{ Greatest primary tumor dimension } \\
\hline$\leq 30$ & 10 & 21 & NS \\
\hline$>30$ & 22 & 25 & \\
\hline \multicolumn{4}{|l|}{ Lymphatic invasion } \\
\hline None to mild & 28 & 39 & NS \\
\hline Moderate/Severe & 4 & 7 & \\
\hline \multicolumn{4}{|l|}{ Vascular invasion } \\
\hline Negative & 28 & 39 & NS \\
\hline Positive & 4 & 7 & \\
\hline
\end{tabular}

Table 3. Correlation between fibrotic focus (FF) and clinicopathological findings

\begin{tabular}{|c|c|c|c|}
\hline \multirow[b]{2}{*}{ Variable } & \multicolumn{2}{|c|}{ Fibrotic focus (FF) } & \multirow[b]{2}{*}{$P$ value } \\
\hline & Positive $(n=29)$ & Negative $(n=49)$ & \\
\hline \multicolumn{4}{|l|}{ Depth of tumor } \\
\hline pT1 & 6 & 25 & 0.008 \\
\hline pT2 & 23 & 24 & \\
\hline \multicolumn{4}{|l|}{ Pathological type } \\
\hline Differentiated & 19 & 27 & NS \\
\hline Undifferentiated & 10 & 22 & \\
\hline \multicolumn{4}{|c|}{ Greatest primary tumor dimension } \\
\hline$\leq 30$ & 8 & 23 & 0.09 \\
\hline$>30$ & 21 & 26 & \\
\hline \multicolumn{4}{|l|}{ Lymphatic invasion } \\
\hline None to mild & 26 & 41 & NS \\
\hline Moderate/Severe & 3 & 8 & \\
\hline \multicolumn{4}{|l|}{ Vascular invasion } \\
\hline Negative & 26 & 41 & NS \\
\hline Positive & 3 & 8 & \\
\hline
\end{tabular}

Tables 2 and 3). FF tended to be positive in metastasis from larger tumors (Table 3 ). There was a strong correlation between ECI and FF $(P=0.001$; Table 4$)$.

\section{Prognosis}

The median follow up was 93 months (range, 2-223 months). Disease recurrence was observed in only ten patients; three had recurrence in the liver and three in distant lymph nodes, three patients had local recurrence, and one had lung metastases. The time interval between surgery and recurrent disease was 10-86 months (mean, 38 months). There were skip metastases at the N2 station in six patients and at the N3 station in one patient, in whom no recurrence was observed.

Disease-free survival (DFS) differed significantly according to ECI and FF; the 10-year DFS rates of
Table 4. Correlation between ECI and FF

\begin{tabular}{lccc} 
& \multicolumn{3}{c}{ FF } \\
ECI & Positive & Negative & $P$ \\
\hline Positive & 25 & 7 & 0.001 \\
Negative & 4 & 42 & \\
\hline
\end{tabular}

patients with and without ECI were $73 \%$ and $90 \%$, respectively, while the 10 -year DFS rates for patients with and without FF were $74 \%$ and $90 \%$, respectively (Figure 4). Recurrence was not significantly associated with AT (Table 5), PP, or IL. By Cox multivariate analysis, venous invasion was a significant independent factor for recurrence $(P=0.02)$, while positive ECI or $\mathrm{FF}$ indicated a trend to recurrence. The incidence of 
Disease free rate ECI

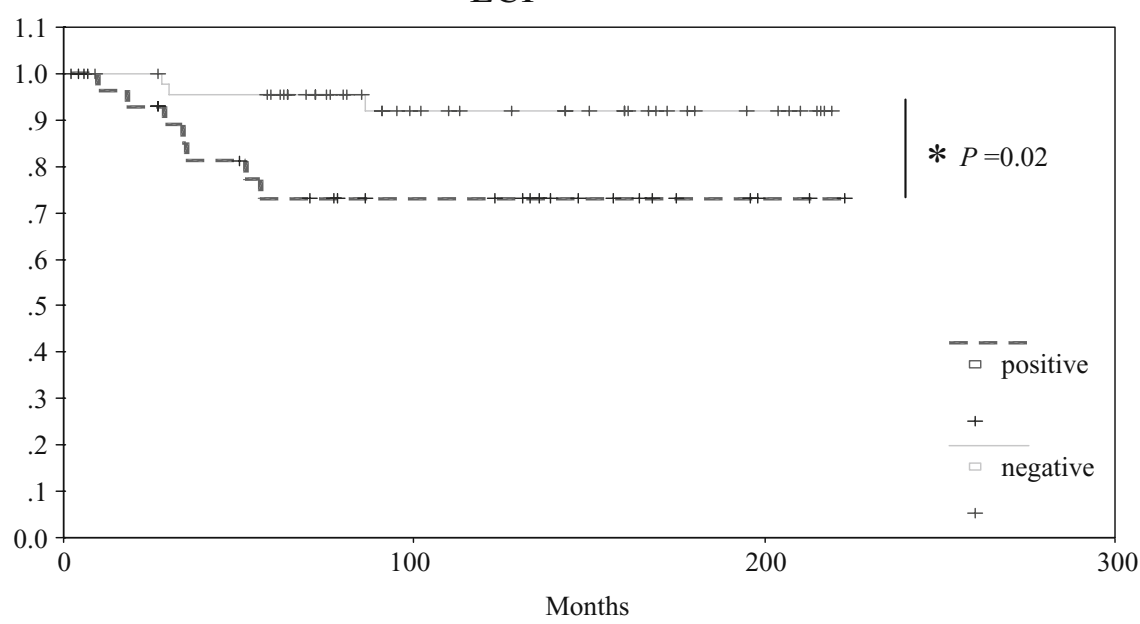

Disease free rate

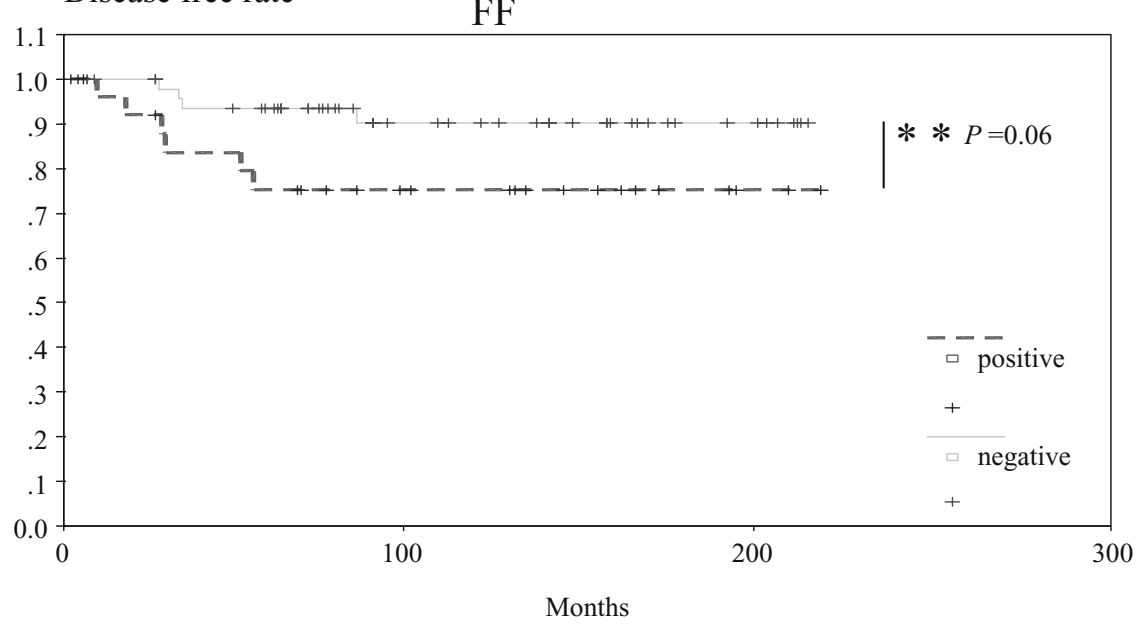

Fig. 4. Disease-free survival by ECI and $\mathrm{FF}$

Table 5. Size of metastatic focus (AT) and recurrence

\begin{tabular}{lcccc}
\hline & \multicolumn{4}{c}{ AT } \\
\cline { 2 - 4 } Recurrence & Macro $(>2 \mathrm{~mm})$ & Micro $(\leq 2 \mathrm{~mm})$ & ITC $(<0.2 \mathrm{~mm})$ & $P$ \\
\hline Positive & 6 & 4 & 0 & 0.8 \\
Negative & 38 & 27 & 3 & \\
\hline
\end{tabular}

ITC, isolated tumor cell

recurrence was not different between patients with subserosal (ss) tumors without ECI /FF and those with submucosal $(\mathrm{sm})$ or muscularis propria $(\mathrm{mp})$ tumors with ECI or FF; recurrence was shown in $14 \%(1 / 7)$ of the former and in $15 \%(2 / 13)$ of the latter.

Similarly, overall survival (OS) differed significantly according to depth of invasion, venous invasion, and the presence or absence of ECI in these patients. The 10year OS rates of patients with and without ECI were 50 $\%$ and $80 \%$, respectively, while the 10 -year OS rates of patients with and without $\mathrm{FF}$ were $50 \%$ and $79 \%$, respectively. Multivariate analyses revealed that ECI or FF was a significant prognosticator $(P=0.01$; Table 6$)$.

\section{Discussion}

Many investigators have reported that, in patients with various malignancies, the pathological characteristics of metastatic nodes could be associated with survival [2-6]: however, some of the populations $[3,6]$ might not be selected properly in the previous studies. In patients 
Table 6. Univariate and multivariate analyses for overall survival

\begin{tabular}{|c|c|c|c|c|}
\hline & \multicolumn{2}{|c|}{ Univariate analysis } & \multicolumn{2}{|c|}{ Multivariate analysis } \\
\hline & Category & $P$ & $P$ & Relative risk \\
\hline Primary tumor size & $>/ \leq 30 \mathrm{~mm}$ & NS & NS & \\
\hline Histology & Differentiated/Undifferentiated & NS & 0.07 & 1.6 \\
\hline Venous invasion & Positive/Negative & 0.05 & NS & \\
\hline Depth of invasion & $\mathrm{pT} 2 / \mathrm{pT} 1$ & 0.03 & NS & \\
\hline Size of metastasis & Macro/Micro & NS & NS & \\
\hline ECI or FF & Positive/Negative & 0.007 & 0.01 & 2.01 \\
\hline Lymphatic invasion & 0 - Mild/Moderate & NS & 0.07 & 1.6 \\
\hline Nodal status & $\mathrm{n} 1 / \mathrm{n} 23$ & NS & NS & \\
\hline
\end{tabular}

with localized gastric cancer, the number of metastatic nodes and the presence of serosal invasion were the strongest prognosticators, the latter being associated with peritoneal metastasis. However, to identify the prognostic impact of nodal findings only, among other strong prognosticators, a study population should be limited to those patients with tumors in which the nodal findings could primarily determine the prognosis. Therefore, in the present study, we included patients with localized gastric tumors which had not extended deeper than the serosal surface and which had metastasized to only a single node (pT1 or pT2).

Of the many histopathological findings of lymph nodes, ECI is considered to be important, because ECI has been reported to be an indicator of poor prognosis, as observed in various cancers $[3,4,6]$. Some authors have suggested that ECI could represent a tumor with high aggressiveness. For gastric carcinoma, Alfred et al. [6] reported that once the capsules of the lymph nodes had been penetrated by tumor tissue, the chances of cure by surgery were minimal. Previously, Tanaka et al. [7] reported that perinodal involvement may be implicated in the development of peritoneal dissemination by lymphatic routes and was a significant risk factor for peritoneal metastasis in patients with $\mathrm{T} 2 / 3$ gastric cancer. Our results indicated that ECI was an independent prognosticator for survival in patients with gastric cancer by selecting patients. We consider that ECI is not suggestive of the route for peritoneal metastasis but that it is suggestive of tumor cell aggressiveness.

Natsugoe et al. [12] indicated that deposits of tumor cells (micrometastasis) found in lymph nodes may be classified as metastatic or nonmetastatic cells with regard to malignant potential. Metastatic tumor deposits are those with stromal reaction, and nonmetastatic tumor deposits are so-called isolated tumor deposits without stromal reaction [12]. We assessed stromal reaction in metastatic lymph nodes by examining FF. An FF was defined as a scar-like area replacing necrosis. In 1998 Hasebe et al. [13] proposed FF as an indicator of tumor aggressiveness in invasive ductal carcinoma of the breast; however, to our knowledge, no study has addressed the prognostic significance of FF within lymph nodes in patients with gastric cancer. Our study demonstrated that DFS was relatively worse $(P=0.06)$ in patients who had an FF in the metastatic lymph node than in those patients without FF, though ECI and FF were strongly associated with each other (Table 5). These findings suggest that stromal cells help intranodal tumor cells to invade extracapsular areas and may play an important role in the development of further metastases.

Although the diagnostic criteria of lymph node metastasis are generally based on whether cancer cells are present or absent, metastatic foci vary in size, from small sinusoidal tumor cell aggregates to large deposits of tumor completely replacing the nodal parenchyma. In breast carcinoma, larger axillary nodal metastasis is reported to be associated with worse prognosis $[14,15]$. It is a problem that there is no consensus on how nodal metastasis should be measured, and many studies provide no details on methodology. Millis et al. [16] addressed the detailed methodology of measurement and suggested that survival in patients with breast cancer was not related to the size of the metastatic foci. We measured the maximum dimension of the metastatic foci and defined micrometastasis as measuring $2 \mathrm{~mm}$ or less and macrometastasis as measuring more than $2 \mathrm{~mm}$. However, the size of the metastatic focus was not associated with the pathological findings of the primary tumors or with prognosis. In 2002, the American Joint Committee on Cancer staging system for breast cancer defined the lower size limit for lymph node micrometastases of breast cancer as $0.2 \mathrm{~mm}$ in diameter and the upper limit as $2 \mathrm{~mm}$ [17]. However, the rationale for applying of this criterion to gastric cancer remains unclear.

There have been several studies of the prognostic significance of IL in breast carcinoma [16, 18], however, most of them showed no significant differences in prognosis according to intranodal location (IL). In gastric carcinoma, no studies have addressed the PP and IL of the metastatic focus. However, our results sug- 
gested that the differences in IL resulted from the histology of the primary tumors and the presence of venous invasion.

Because we focused on a single metastasis, it seemed appropriate to investigate the biology of the sentinel lymph node [19]. As in previous reports, we also found some skip metastases in the second- and third-tier lymph node stations, but the location was not relevant to prognosis. We did, however, clarify the prognostic value of the nodal histology in patients with a few confounding prognostic factors. It was found that ECI or FF was prognostic in pT1/2 cancer with a single metastasis, whereas AT, PP, and IL were not prognostic factors. In conclusion, it is considered that the presence of ECI or $\mathrm{FF}$ is an independent prognosticator for relatively early gastric cancer. Further prospective studies are necessary to evaluate the real role of these stromal reactions in the development of metastasis.

\section{References}

1. Yoshikawa T, Tsuburaya A, Kobayashi O, Sairenji M, Miyagi Y. Protein levels of tissue inhibitor of metalloproteinase-1 in tumor extracts as a marker for prognosis and recurrence in patients with gastric cancer. Gastric Cancer 2006;9:106-13.

2. Cai J, Ikeguchi M, Maeta M, Kaibara N, Sakatani T. Clinicopathological value of immunohistochemical detection of occult involvement in pT3N0 gastric cancer. Gastric Cancer 1999;2: 95-100.

3. Giorgio A, Botti C, Sammartino P, Mingazzini P, Flammia M, Stipa V. Extracapsular lymph node metastases in the staging and prognosis of gastric cancer. Int Surg 1991;76:218-22.

4. Leonard C, Corkil M, Tompkin J, Zhen B, Waitz D, Norton L, Kinzie J. Are axillary recurrence and overall survival affected by axillary extranodal tumor extension in breast cancer? Implications for radiation therapy. J Clin Oncol 1995;13:47-53.

5. Komuta K, Okudaira S, Haraguchi M, Furui J, Kanematsu T. Identification of extracapsular invasion of the metastatic lymph nodes as a useful prognostic sign in patients with resectable colorectal cancer. Dis Colon Rectum 2001;44:1838-44.

6. Alfred Z, Christian C, Kaj F. Surgical treatment of gastric malignancies. Ann Surg 1974;179:94-101.
7. Tanaka T, Kumagai K, Shimizu K, Masuo K, Yamagata K. Peritoneal metastasis in gastric cancer with particular reference to lymphatic advancement; extranodal involvement is a significant risk factor for peritoneal metastasis. J Surg Oncol 2000;75: 165-71.

8. Koike H, Ichikawa D, Kitamura K, Tsuchihashi Y, Yamagishi H. Perinodal involvement of cancer cells in gastric cancer patients. Surgery 2004;135:266-72.

9. Etoh T, Sasako M, Ishikawa K, Katai H, Shimoda T. Extranodal metastasis is an indicator of poor prognosis in patients with gastric carcinoma. Br J Surg 2006;93:369-73.

10. Japanese Gastric Cancer Association. Japanese classification of gastric carcinoma 2nd English edition. Gastric Cancer 1998;1: $10-24$.

11. Arai K, Iwasaki Y, Takahashi T. Clinicopathological analysis of early gastric cancer with solitary lymph node metastasis. Br J Surg 2002;89:1435-7.

12. Natsugoe S, Aikou T, Shimada M, Yoshinaka H, Takao S, Shimazu H, Matsushita Y. Occult lymph node metastasis in gastric cancer with submucosal invasion. Jpn J Surg 1994;24:870-5.

13. Hasebe T, Tsuda H, Hirohashi S, Shimosato Y, Tsubono $\mathrm{Y}$, Yamamoto H, Mukai K. Fibrotic focus in infiltrating ductal carcinoma of the breast: a significant histopathologic parameter for predicting the long-term survival of the patients. Breast Cancer Res Treat 1998;49:195-208.

14. Huvos AG, Hutter R, Berg JW. Significance of axillary macrometastases and micrometastases in mammary cancer. Ann Surg 1971;173:44-6.

15. Fan Y, Tan Y, Wu C, Treseler P, Lu Y, Chan C, et al. The effect of sentinel node tumor burden on non-sentinel nodes status and recurrence rates in breast cancer. Ann Surg Oncol 2005;12: 705-11.

16. Millis RR, Springall R, Lee AHS, Ryder K, Rytina ERC, Fentiman ISF. Occult axillary lymph node metastases are of no prognostic significance in breast cancer. Br J Cancer 2002; 86:396-401.

17. Singletary SE, Allred C, Ashley P, Bassett LW, Berry D, Kirby I, et al. Revision of the American Joint Committee on Cancer staging system for breast cancer. J Clin Oncol 2002;20:3628-36.

18. McGuckin MA, Cummings MC, Walsh MD, Hohn BG, Bennett IC, Wright RG, et al. Occult axillary node metastases in breast cancer; their detection and prognostic significance. Br J Cancer 1996;73:88-95.

19. Tsuburaya A, Noguchi Y, Yoshikawa T, Kobayashi O, Sairenji M, Motohashi H. Solitary lymph node metastasis of gastric cancer as a basis for sentinel lymph node biopsy. Hepatogastroenterology 2002;49:1449-52. 